\section{The significance of the squeeze test to identify arthritis was underestimated or not?}

We read with deep interest the article by van den Bosch $e t a l^{1}$ related to the diagnostic accuracy of the squeeze test to identify arthritis. This cross-sectional cohort study suggested that a positive squeeze test is associated with local joint inflammation with a very low sensitivity, indicating a high percentage of swollen joints with a negative squeeze test. Doctors should 'keep in mind' the characteristics of this test when performing in daily practice. We really appreciate the work that has been done by the authors. The findings help clinicians understand the effect of the squeeze test to identify arthritis. However, there are some worthwhile issues that need to be explored.

The squeeze test is an easy, rapid and cheap method to identify arthritis in metacarpophalangeal (MCP) and metatarsophalangeal joints for general practitioners (GPs) as promoted by some experts and societies. ${ }^{2-4}$ We agree with the authors' point of view that this screening test requires a high sensitivity to prevent false negatives. However, we are confused about the inclusion criteria. We are confused that the authors excluded patients with arthralgia who had been diagnosed as 'apparent arthritis' or 'no arthritis' at first visit, since all these patients were referred by GPs due to 'suspected arthritis'. The squeeze test might be more accurate to diagnose these patients, which would obviously increase the sensitivity of the test. The authors also didn't illustrate whether there is a 'squeeze test'-based referral by the GPs. These might affect either the sensitivity or the specificity of the squeeze test.

Aside from the inclusion criteria, we are also confused about the assessments. Evidence indicates that early aggressive treatment results in greater improvement than therapy initiated later in rheumatoid arthritis (RA) disease. ${ }^{5}{ }^{6}$ It's very important that GPs could identify patients with suspected RA and refer them to rheumatological experts. The squeeze test could help GPs for making a rapid diagnosis of 'clinical suspicion' of RA in the primary care population. Is it equitable that authors used this test to 'identify arthritis or swollen joints' and evaluated its value? As mentioned by authors, study showed a poor correlation of MRI-detected inflammation with joint swelling at physical examination, and bone marrow oedema and joint tenderness occurred frequently in clinically non-swollen joints. ${ }^{7}$ Even swollen joints vary in different types of arthritis, which means the results of squeeze test between RA and other non-inflammatory musculoskeletal arthritis are different. Furthermore, the test of both hands and foots will be more sensitive to identify suspected RA for its bilateral affection, in contrast to any other arthritis. So, we have no idea whether it is appropriate to assess the value of squeeze test by one hand and one foot for each patient.

In addition to all of the above, there are some other issues that need to be mentioned. First, the authors added proximal interphalangeal joints (PIPs) symptoms as positive physical examination, but there lacked the results of squeeze test about PIPs. Second, 'the squeeze test' described by the authors in figure $1^{1}$ isn't the 'validated standard squeeze test' promoted by experts and societies. ${ }^{2-4}$ The interphalangeal joints of the hands and feet are held by hand and their pain will lead to a false-positive squeeze test. Third, the greatest advantage of the squeeze test is that it is easy and cheap. The GPs can repeat this test in a few weeks to make sure they never miss a suspicion, which could obviously increase the sensitivity of this test to identify RA, since the authors indicated that the positive squeeze test of MCP in patients with clinical suspicion for progression to arthritis is very low. We are also very curious about the relationship between two more repeat squeeze tests and MRI-detected inflammation as outcome. Finally, we didn't find the numbers of positive/negative squeeze tests in positive/negative physical examination groups as well as the control group in manuscript or supplementary tables.

We respect the great contributions of the authors and we would also be very much interested in the authors' response to these issues.

\section{Wei Luo, Chao Zeng, Hongbo He}

Department of Orthopaedics, Xiangya Hospital, Central South University, Changsha, Hunan Province, China

Correspondence to Professor Hongbo He, Department of Orthopaedics, Xiangya Hospital, Central South University, Xiangya Road, Changsha, Hunan Province 410008, China; 88569810@qq.com

To cite van den Bosch WB, Mangnus L, Reijnierse M, et al. The diagnostic accuracy of the squeeze test to identify arthritis: a cross-sectional cohort study. Ann Rheum Dis 2015.

Twitter Follow Hongbo He at@88569810@qq.com

Competing interests None declared.

\section{Patient consent Obtained.}

Provenance and peer review Not commissioned; internally peer reviewed.

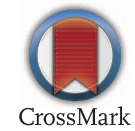

To cite Luo W, Zeng C, He H. Ann Rheum Dis 2015;74:e60.

Received 13 July 2015

Accepted 14 July 2015

Published Online First 31 July 2015

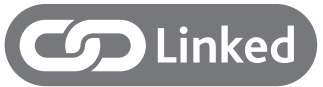

http://dx.doi.org/10.1136/annrheumdis-2015-208236

Ann Rheum Dis 2015;74:e60. doi:10.1136/annrheumdis-2015-208221

\section{REFERENCES}

1 van den Bosch WB, Mangnus L, Reijnierse M, et al. The diagnostic accuracy of the squeeze test to identify arthritis: a cross-sectional cohort study. Ann Rheum Dis 2015;74:1886-9.

2 http://www.nras.org.uk/2-first-visit-to-gp (accessed 25 Nov 2014).

3 Emery P, Breedveld FC, Dougados $M$, et al. Early referral recommendation for newly diagnosed rheumatoid arthritis: evidence based development of a clinical guide. Ann Rheum Dis 2002;61:290-7.

4 Wiesinger T, Smolen JS, Aletaha D, et al. Compression test (Gaenslen's squeeze test) positivity, joint tenderness, and disease activity in patients with rheumatoid arthritis. Arthritis Care Res (Hoboken) 2013;65:653-7.

5 Gremese E, Salaffi F, Bosello SL, et al. Very early rheumatoid arthritis as a predictor of remission: a multicentre real life prospective study. Ann Rheum Dis 2013;72:858-62.

6 Puolakka K, Kautiainen H, Mottonen T, et al. Early suppression of disease activity is essential for maintenance of work capacity in patients with recent-onset rheumatoid arthritis: five-year experience from the FIN-RACo trial. Arthritis Rheum 2005;52:36-41.

7 Krabben A, Stomp W, Huizinga TW, et al. Concordance between inflammation at physical examination and on MRI in patients with early arthritis. Ann Rheum Dis 2015;74:506-12. 